ARTÍCULO ORIGINAL
Rev Colombiana Cienc Anim 2017; 9 (Supl):67-75.

\title{
Buenas prácticas ganaderas en hatos lecheros de Santa Rosa de Cabal, Risaralda, Colombia
}

\author{
Good livestock practices in dairy farms in Santa Rosa de Cabal, Risaralda, \\ Colombia
}

\author{
Marín A, Libia ${ }^{1}$ M.Sc, Arredondo B, Julia ${ }^{1 *}$ PhD, Hernandez H, Darwin ${ }^{2}$ PhD.
}

${ }^{1}$ Corporación Universitaria Santa Rosa de Cabal. Facultad de Ciencias Pecuarias. Grupo de investigación en reproducción y mejoramiento genético. Santa Rosa de Cabal, Risaralda. ${ }^{2}$ Universidad de Sucre - Campus Ciencias Agropecuarias. Departamento de Zootecnia. Facultad de Ciencias Agropecuarias. Sincelejo. Colombia.

Keywords:

Hygiene;

Quality;

Safety;

Milk production systems

Palabras Clave:

Calidad;

Higiene;

Inocuidad;

Sistemas de producción de leche

\begin{abstract}
The objective of the present study was to analyze the degree of compliance of these practices, by evaluation of 99 variables in 21 productive systems in Santa Rosa de Cabal, Risaralda. Only one of the farms complied with the 48 fundamental criteria required and, the degree of compliance of major and minor criteria was insufficient. Only 8 of the farms are certified as free of brucellosis and tuberculosis. The best performing areas are animal welfare $(81 \%)$ and those related to the milking routine $(79.1 \%)$; On the other hand, the most deficient were the control of veterinary drugs and agricultural products $(5.8 \%)$, as well as the conditions of the cooling tank (32.6\%). Through a Multiple Correspondence Analysis, a significant association was found between variables involved with the guarantee in the safety of the milk, regarding the operation, cleaning and disinfection of equipment that guarantee an adequate temperature. There was also an association between variables involved in the milking procedure and hygienic conditions during the milking procedure, involving both the operator and the animal. A lack of training and awareness about the importance of compliance with many of these criteria was found, this is a fundamental tool to obtain a diagnosis of the current sanitary status, and in which it may be necessary to take into account other variables, not included in the current checklist, for a later implementation and follow-up to improvement plans of the productive systems.
\end{abstract}

\section{Resumen}

El objetivo del presente trabajo fue analizar el grado de cumplimiento de BPG, mediante la evaluación de 99 variables en 21 sistemas productivos del municipio de Santa Rosa de Cabal, Risaralda. Solo uno de los predios cumplió con los 48 criterios fundamentales exigidos y en general, el grado de cumplimiento de criterios mayores y menores fue insuficiente. Solo 8 de los hatos están certificados como libres de brucelosis y tuberculosis. Las áreas de mejor desempeño son el bienestar animal $(81 \%)$ y las relacionadas con la rutina de ordeño $(79,1 \%)$; en cambio, las mas deficientes fueron el control de medicamentos veterinarios e insumos agropecuarios (5,8\%), así como las condiciones del tanque de enfriamiento (32,6\%). Mediante unAnálisis de Correspondencia múltiple, se encontró asociación significativa entre variables que tienen que ver con la garantía en la inocuidad de la leche, en lo referente a la operación, limpieza y desinfección de equipos que garanticen una temperatura adecuada. También hubo asociación entre variables que tienen que ver con el procedimiento de ordeñoy condiciones de higiene durante la faena, que involucran tanto al operario, como al animal. Se evidencia falta de capacitación y concientización acerca de la importancia del cumplimiento de muchos criterios de BPG, que es una herramienta fundamental para obtener un diagnostico del estado actual del hato, y en la cual posiblemente sea necesario tener en cuenta otras variables, no incluidas en la lista de verificación actual, para una posterior implementación y seguimiento a planes de mejoramiento de los sistemas productivos.
Recibido: 18-11-2016;

Aceptado: 29-04-2017.

Correspondencia autor:

victoria.arredondo@unisarc.edu.co 


\section{INTRODUCCIÓN}

Las Buenas Prácticas Ganaderas (BPG), están reguladas en Colombia por el decreto 616 del 28 de febrero de 2006 del Ministerio de la Protección Social, el Ministerio de Agricultura y Desarrollo Rural, y por la Resolución 3585 del 2008 del Instituto Colombiano Agropecuario (ICA), quienes establecen el reglamento técnico sobre los requisitos que debe cumplir la leche para consumo humano que se obtenga, procese, envase, transporte, comercializa, expenda, importe o exporte en el país (MINISTERIO DE SALUD Y PROTECCIÓN SOCIAL, 2013).

Dicho reglamento regula las actividades necesarias para la obtención de leche en los diferentes modelos de producción ganadera; su cumplimiento es importante porque según lo establecido en las normas sanitarias de alimentos, la leche y sus derivados lácteos están considerados como alimentos de alto riesgo en salud pública y por lo tanto, deben cumplir con los requisitos que se establezcan para garantizar la vida, salud y seguridad humana (MINISTERIO DE PROTECCIÓN SOCIAL, 2006).

Su aplicación se direcciona hacia la obtención eficiente de leche inocua y de calidad, y debido a que establecen requerimientos para lograr producciones que no afecten al medio ambiente, cuiden la salud de los trabajadores y contribuyan al bienestar animal, su cumplimiento es fundamental (VILLOCH, 2010). Así que los sistemas deben ser cada vez más competitivos, atendiendo a las exigencias crecientes del mercado, con el fin de ser sostenibles en el tiempo. Su monitoreo, permite evaluar la forma de producción y mejorar constantemente la actividad de los sistemas. Sin embargo, han sido estigmatizadas como un proceso complicado, inalcanzable, inviable y poco importante, lo que se ha corroborado con el escaso número de predios certificados. Tal percepción obedece a la poca divulgación, capacitación y apoyo por parte de las entidades estatales y privada (MUR y MOLANO, 2016).

En el departamento de Risaralda, el municipio de Santa Rosa de Cabal ocupa el segundo lugar en producción ganadera, dado que su lechería especializada representa más del $12 \%$ de la producción de leche a nivel departamental. El objetivo esta investigación fue analisar el grado de cumplimiento de BPG en 21 hatos lecheros del municipio de Santa Rosa de Cabal (Risaralda), con el fin de evaluar los aspectos más críticos, así como los de mayor eficiencia, planteando un panorama del estado actual de los sistemas productivos en el municipio.

\section{MATERIALES Y MÉTODOS}

Mediante un muestreo aleatorio, se obtuvo información de 21 hatos lecheros del municipio de Santa Rosa de Cabal. Mediante entrevista a los productores y tomando como referencia la lista de verificación implementada en la resolución 3585 de 2008 del Instituto Colombiano Agropecuario (ICA), se obtuvo información correspondiente a 99 variables cualitativas, que fueron codificadas en categoría ordinal, correspondientes a las siguientes áreas: sanidad animal y bioseguridad (16), manejo integrado de plagas (4), registros y documentación (3), suministro y calidad del agua (3), bienestar animal (4), condiciones del cuarto de tanque de enfriamiento (13), sistema de ordeño y sitio de ordeño (9), rutina de ordeño (8), protección contra contaminación de la leche (2), manejo de la leche anormal (3), control de medicamentos veterinarios e insumos agropecuarios (20), utensilios y equipos para la faena de ordeño (5), personal (5) y otras, como ubicación, limpieza e identificación de otras áreas (4).

Los datos fueron tabulados en Excel, se realizó un análisis de frecuencias y mediante el software Stata ver. 11.1, se hizo un análisis de correspondencia múltiple para encontrar los factores que encontrar conjuntos de sistemas con determinadas tipologías y determinar el grado de asociación entre las categorías de las variables, explicando toda la información en un número menor de dimensiones o factores.

\section{RESULTADOS Y DISCUSIÓN}

Información general de los sistemas: Se evaluaron 21 sistemas productivos ubicados entre 1550 y 3800 msnm, con 53,2 animales en promedio y una producción promedio de $12 \pm 5,5$ L/vaca/día. El $38,1 \%$ de ellos conformados por cruces de diferentes razas, el $28,6 \%$ por animales de las razas Holstein y Jersey, el $19 \%$ Girolando y el $14,3 \%$, solo raza Holstein.

Con respecto a la distribución del hato, el 52,4\% de los sistemas posee un porcentaje de más del $17 \%$ de vacas secas y el $19 \%$ de ellos tienen todos los animales en producción.

Todos los sistemas poseen pastoreo rotacional, con un predio bien delimitado y animales correctamente identificados en el $95,24 \%$ de los casos. El $34 \%$ de ellos realiza ordeño mecánico $(24 \%$ en potrero y $10 \%$ en sala) y el restante $67 \%$, ordeño manual ( $48 \%$ en potrero y $19 \%$ en sala). Solo en el $81 \%$ de los casos, la localización corresponde al plan de ordenamiento territorial (POT) del municipio. 
Cumplimiento general de criterios: con respecto a la lista de verificación implementada, solo uno de los predios cumplió los 48 criterios fundamentales exigidos para certificación en BPG; en general, se cumplieron entre 15 y 38 de ellos. De los criterios mayores, solo uno de los sistemas sobrepasó con el cumplimiento de 38 criterios, el $52,4 \%$ cumplieron entre 20 y 30 de los 34 mínimos y el $38 \%$ cumplió entre 10 y 20 de ellos. En cuanto a los criterios menores, el $19 \%$ de los sistemas cumplió los 8 criterios mínimos y en general, el cumplimiento fue entre 2 y 10 de dichos criterios.

Para que un hato sea certificado en BPG, es necesario el cumplimiento del $100 \%$ de los criterios fundamentales, al menos el $85 \%$ de los mayores y el $60 \%$ de los menores, según el manual de Inspección adoptado en el artículo 18 de la resolución ICA 3585 de 2008 , obteniendo así una bonificación de 0,35 centavos de dólar, por litro de leche, cuando es comercializada a acopiadoras nacionales como Alpina, Alival, Colanta, Celema, entre otras (MADR, 2012).

Solo 8 de los 21 hatos contaron con certificación oficial como libres de brucelosis y tuberculosis, otorgada por ICA, basada en las resoluciones 1332 del 2013 y 1332 del 2015 respectivamente (ICA, 2013 y 2015), en las cuales se enfatiza la importancia de dichas enfermedades, por su carácter zoonótico y su alto impacto económico en el sector ganadero del país.

El cumplimiento de BPG, además de los beneficios económicos, da organización a la empresa ganadera, tanto en aspectos estructurales (instalaciones) y manejo (bienestar animal y humano), como gerenciales y económicos. Aunque la verdadera importancia de alcanzar esta certificación radica en convertir a Colombia en un país competitivo, más aún con los inequitativos tratados de libre comercio firmado (MUR y MOLANO, 2016).

Las áreas en las cuales los productores cumplieron mayor número de criterios en promedio, son el bienestar animal (81\%) y las relacionadas con la rutina de ordeño $(79,1 \%)$; en cambio, en áreas como el control de medicamentos veterinarios e insumos agropecuarios, así como las condiciones del tanque de enfriamiento, los productores solo cumplen con el 5,8 y $32,6 \%$ de los criterios, respectivamente.

Criterios como adecuadas condiciones para el manejo animal y la disponibilidad de agua y alimento, son cumplidos por todos los productores.

Sanidad y bienestar animal, manejo de medicamentos e insumos y bioseguridad: los productores cumplieron con el $49,1 \%$ de los criterios.
Se destacan la adecuada delimitación del predio e identificación de los animales en el 95,24\% de los casos y la vacunación vigente contra las enfermedades de control oficial ( $85,71 \%$ de ellos). Aunque Colombia ha sido declarada como libre de fiebre aftosa con vacunación, la admisibilidad a los mercados internacionales puede estar limitada por otros enfermedades como la brucelosis (prevalencia, $>4 \%$ ) o la tuberculosis (CARULLA y ORTEGA, 2016). Los productores que no cuentan con el Certificado Único de Vacunación (CUV), no pueden acceder a guías de movilización para transporte de los semovientes, por lo cual deben realizar esta actividad, así como la comercialización de la leche, de manera clandestina, convirtiéndose en un foco de alto riesgo para la proliferación deenfermedades tanto de control oficial como de no control.

Solo el $9,52 \%$ de los productores realiza cultivos y antibiogramas cuando se presentan eventos como la mastitis, que es, con la cojeras e hipocalcemia, uno de los problemas sanitarios más comunes y cuyo manejo tiene un componente cultural importante, según el cual, se usan productos recomendados en predios contiguos o en almacenes veterinarios.

En el área de bienestar animal, criterios como una adecuada disposición y disponibilidad de agua y alimento, así como condiciones para el manejo animal, son adecuadamente cumplidos por la totalidad de los productores.

El $76,19 \%$ posee tanque de almacenamiento de agua, aunque solo el $42,85 \%$ monitorea su calidad, y de ellos solo el $19,04 \%$ aplica acciones correctivas frente a un determinado hallazgo.

Con respecto a la realización de intervenciones quirúrgicas, que son esporádicas, y las no quirúrgicas, como topización, marcación y aplicación de medicamentos, solo son documentadas en el 33,33\% de los casos.

El control de medicamentos veterinarios e insumos agropecuarios fue una de las áreas en las que los productores cumplieron el menor número promedio de criterios. Sin embargo, el 90,5\% tiene todos los productos dentro de su periodo de vigencia y el $85,7 \%$ cuenta con instrumentos adecuados para suadministración, maneja medicamentos con registro ICA y usa suplementos en la alimentación animal.

Con respecto a las áreas e instalaciones sanitarias, menos de la mitad $(47,62 \%)$ cuenta con una apropiada identificación de áreas y solo el $57,14 \%$ posee instalaciones sanitarias dentro del sistema. 
Aproximadamente en la mitad de los casos $(47,62 \%)$, se da un manejo adecuado a las basuras, el estiércol de las instalaciones y los residuos peligrosos; el $42,85 \%$ implementa acciones para el control de plagas y solo el $38,09 \%$ clasifica las basuras.

Registros y documentación: El 19\% de los productores no cumplió con ninguno de los criterios correspondientes esta área. El $66,7 \%$ de los productores tiene un registro o ficha individual de cada animal, pero solo el $28,57 \%$ de ellos mantiene un archivo vigente con todos los registros. La causa más común, según los productores, es la falta de tiempo para registrar eventos, las complicaciones posteriores pueden están relacionadas con desconocimiento del momento óptimo de eventos reproductivos (servicio, parto), productivos (días de lactancia, producción por lactancia) y de la rentabilidad, además de los escasos criterios para seleccionar animales superiores en el hato.

El 61,9\% maneja guías sanitarias de movilización, que en Colombia se rigen por la Resolución 3278 de 2008 (ICA, 2008), y son un instrumento sanitario de control epidemiológico que se expide para el o los animales a movilizar y permiten prevenir la difusión de enfermedades y la ocurrencia de epidemias.

Los riesgos asociados a la movilización ilegal son traslado de animales vacunados o positivos a pruebas de laboratorio, a zonas libres (sin vacunación), traslado de animales de contrabando, mezclas de animales de diferentes orígenes, y reemplazo por animales enfermos o infectados (ICA, 2013).

Personal: el $71,43 \%$ de los sistemas tiene a sus empleados inscritos en el sistema general de seguridad social en salud, alrededor de la mitad $(57,14 \%)$ cuenta con un botiquín y al menos una persona que haya realizado curso de primeros auxilios y solo el $23,81 \%$ posee un programa de capacitación soportado por certificados de asistencia.

Manejo del ordeño: Fue una de las áreas en las cuales se encontró mayor grado de implementación por parte de los productores. El $95,24 \%$ de los productores implementa una eficiente limpieza de pezones durante el ordeño, en el cual además, se utilizan equipos y utensilios de materiales adecuados. El 90,47\% realiza un adecuado sellado y mantiene flancos, ubre y cola de los animales limpios en el momento de ordeño; el $80,95 \%$ realiza adecuadamente tanto despunte, como secado y desinfección (en el 76,19\% de los casos) de pezones.

En el $90,48 \%$ de los casos, el ordeño es realizado en un sitio apropiado y exclusivo para tal fin, con mangueras de agua en buen estado $(85,71 \%)$, buena ventilación
$(80,95 \%)$, condiciones adecuadas de higiene en la zonade espera y un manejo de residuos sólidos y líquidos inocuo para fuentes de agua, ambiente y proliferación de plagas en el $71,43 \%$ de los sistemas.

A pesar de ello, solo el $28,57 \%$ cuentan con un procedimiento de limpieza y desinfección para el sitio de ordeño y en más de la mitad de los casos $(57,14 \%)$ la sala de ordeño no cuenta con restricción para otro tipo de animales. El ingreso de otros animales al sitio de ordeño, específicamente la presencia de materia fecal, es una fuente de contaminación de alto riesgo para la leche y puede llegar a ocurrir ingestión accidental por parte de los bovinos, generando problemas de endoparásitos (MINISTERIO DE LA PROTECCIÓN SOCIAL, 2006; ICA, 2008).

Solo el $66,7 \%$ de los sistemas exigen a los ordeñadores lavado de manos y antebrazos antes de iniciar la rutina de ordeño y el $47,61 \%$ no cuenta con un procedimiento para la rutina de ordeño, solo el $42,86 \%$ cuenta con procedimientos de limpieza y desinfección para equipos y utensilios y el $80,95 \%$ reutiliza los filtros para la leche y los almacena de forma inadecuada. El registro de mantenimiento preventivo de equipos de ordeño solo se encontró en el $19,05 \%$ de los casos.

De los productores que cuentan con tanque de enfriamiento, cerca de la mitad $(47,61 \%)$ tiene las mangueras de agua en adecuado estado y el $42,86 \%$ efectúa protocolos de limpieza y desinfección. El $38,09 \%$ utiliza el cuarto del tanque únicamente para los propósitos establecidos, garantiza temperatura por debajo de $6^{\circ} \mathrm{C}$ y mantiene en buen estado el equipo de refrigeración. Se destaca el bajo porcentaje $(19,04 \%)$ de productores que implementan pisos, paredes y techos debidamente acabados y con superficies fáciles de limpiar y desinfectar y que tienen además drenajes con sifón o trampa que impidan el acceso de plagas al área.

Con respecto al manejo de la leche anormal, correspondiente a la leche en retiro por aplicación de antibióticos $u$ otros medicamentos, presencia de mastitis clínica con alto recuento de células somáticas y unidades formadoras de colonia o por presencia de sangre, se destaca el alto porcentaje de productores que no cumple con ningún criterio en esta área $(19,04 \%)$. Solo el $71,43 \%$ ordeña por separado a las vacas que producen leche anormal, el $76,19 \%$ implementa adecuadamente procedimientos de limpieza de utensilios en contacto con ésta y el $66,66 \%$ la separa y dispone en pozo séptico. Este producto debe ir a sifón y nunca ser homogeneizado con la leche para consumo, debido a los riesgos que ello puede traer para la salud pública

Siendo el precio uno de los principales incentivos para los productores, en algunas zonas del país, 
los acopiadores informales pagan un valor por litro de leche muy similar al pagado por los acopiadores formales, quienes además exigen el cumplimiento de la normatividad anteriormente mencionada, lo que desestimula al productor.

Análisis de correspondencia múltiple. Para el análisis de correspondencia múltiple se tuvieron en cuenta las siguientes variables (Tabla 1 ):
El análisis de correspondencia múltiple mostró que la interrelación entre los factores estudiados explica el $39,59 \%$ de la variabilidad de los datos.

Con respecto a las variables, en el plano factorial, sobre el eje horizontal que representa la primera dimensión, la cual explica el $27,78 \%$ de la variabilidad, se puede observar una asociación significativa entre variables que tienen que ver con la garantía en la inocuidad del producto final que es la leche, dichas variables

Tabla 1. Variables utilizadas en el análisis de correspondencia múltiple

\begin{tabular}{|c|c|c|c|}
\hline Variable & Denominación & Variable & Denominación \\
\hline $\begin{array}{l}\text { Constancia de inscripción de predio } \\
\text { ante la oficina local del ICA }\end{array}$ & $\begin{array}{c}\text { ins1= presencia de certificación, ins2= ausencia } \\
\text { de certificación }\end{array}$ & $\begin{array}{l}\text { Acciones correctivas respecto a la } \\
\text { calidad de agua }\end{array}$ & $\begin{array}{l}\text { cc1 } 1=\text { se toman acciones correctivas } \\
\text { cc2 }=\text { no se toman acciones correctivas }\end{array}$ \\
\hline $\begin{array}{l}\text { Certificación oficial vigente que } \\
\text { acredite el hato como libre de } \\
\text { brucelosis }\end{array}$ & $\begin{array}{c}\text { lb1= existe certificación vigente como libre de } \\
\text { brucelosis } \\
\text { lb2=no existe certificación vigente como libre } \\
\text { de brucelosis }\end{array}$ & Registro ICA & $\begin{array}{l}r 1=\text { se cuenta con registro ICA } \\
\mathrm{r} 2=\text { no se cuenta con registro ICA }\end{array}$ \\
\hline $\begin{array}{l}\text { Certificación oficial vigente que } \\
\text { acredite el hato como libre de } \\
\text { tuberculosis }\end{array}$ & $\begin{array}{c}\text { It1= existe certificación vigente como libre de } \\
\text { tuberculosis } \\
\text { si, It2= no existe certificación vigente como libre } \\
\text { de tuberculosis }\end{array}$ & $\begin{array}{l}\text { Vigencia de los insumos } \\
\text { agropecuarios (IA) }\end{array}$ & $\begin{array}{c}\text { saa } 1=I A \text { vigentes } \\
\text { saa2 }=I A \text { no vigentes }\end{array}$ \\
\hline $\begin{array}{l}\text { Vacunación vigente contra } \\
\text { enfermedades de control oficial }\end{array}$ & $\begin{array}{l}\text { vc1= Vacunación vigente } \\
\text { vc2= Vacunación no vigente }\end{array}$ & $\begin{array}{l}\text { Almacenamiento y transporte de } \\
\text { productos biológicos }\end{array}$ & $\begin{array}{l}\text { at } 1=\text { adecuado almacenamiento y transporte } \\
\text { at } 2=\text { inadecuado almacenamiento y transporte }\end{array}$ \\
\hline $\begin{array}{l}\text { Programa de prevención y control } \\
\text { de mastitis bovina }\end{array}$ & $\begin{array}{l}\mathrm{cms} 1=\text { prevención y control de mastitis bovina } \\
\mathrm{cms} 2=\text { noprevención y control de mastitis bovina }\end{array}$ & $\begin{array}{l}\text { Manejo de medicamentos de } \\
\text { control especial }\end{array}$ & $\begin{array}{c}\text { mc1=Adecuado manejo de medicamentos de } \\
\text { control especial } \\
\text { mc2= Inadecuado manejo de medicamentos de } \\
\text { control especial }\end{array}$ \\
\hline Delimitación del predio & $\begin{array}{l}\mathrm{d} \mid 1=\text { existe delimitación } \\
\mathrm{d} \mid 2=\text { no existe delimitación }\end{array}$ & $\begin{array}{l}\text { Persona responsable para el } \\
\text { manejo de los medicamentos y } \\
\text { biológicos veterinarios (MBV) }\end{array}$ & $\begin{array}{l}\text { mb1= Hay una persona responsable de MBV } \\
\text { mb2=no hay una persona responsable de MBV }\end{array}$ \\
\hline Manejo de animales enfermos & $\begin{array}{l}\text { en1= adecuado manejo de animales enfermos } \\
\text { en2= inadecuado manejo de animales enfermos }\end{array}$ & $\begin{array}{l}\text { Prescripción veterinaria de los } \\
\text { medicamentos }\end{array}$ & $\begin{array}{l}\text { pv1 }=\text { si prescripción veterinaria } \\
\text { pv2=no prescripción veterinaria }\end{array}$ \\
\hline $\begin{array}{l}\text { Instrucciones de manejo sanitario } \\
\text { sobre enfermedades de control } \\
\text { oficial }\end{array}$ & $\begin{array}{c}\text { ime1= se cuenta con instrucciones de manejo } \\
\text { sanitario } \\
\text { ime2=no se cuenta con instrucciones de } \\
\text { manejo sanitario }\end{array}$ & $\begin{array}{l}\text { Respeto del tiempo de retiro de } \\
\text { medicamentos veterinarios }\end{array}$ & $\begin{array}{l}\text { tre } 1=\text { si respeto del tiempo de retiro } \\
\text { tre } 2=\text { no respeto del tiempo de retiro }\end{array}$ \\
\hline Identificación de los animales & $\begin{array}{l}\text { i1= Identificación de los animales } \\
\text { i2=no identificación de los animales }\end{array}$ & Manejo de potreros & $\begin{array}{l}\mathrm{m} 1=\text { hay manejo de potreros } \\
\mathrm{m} 2=\text { no hay manejo de potreros }\end{array}$ \\
\hline Plan sanitario & $\begin{array}{l}\mathrm{p} 1=\text { hay plan sanitario } \\
\mathrm{p} 2=\text { no hay plan sanitario }\end{array}$ & $\begin{array}{c}\text { Registros de aplicación de } \\
\text { medicamentos veterinarios (MV) }\end{array}$ & $\begin{array}{l}\text { ra1= hay registros de aplicación de } \mathrm{MV} \\
\text { ra2=no hay registros de aplicación de MV }\end{array}$ \\
\hline Protocolo adquisición de animales & $\begin{array}{c}\text { ad1=protocolo adquisición externa } \\
\text { ad2=noprotocolo adquisición externa } \\
\text { ad3=autoreemplazos }\end{array}$ & $\begin{array}{l}\text { Acciones correctivas cuando se } \\
\text { detecta el incumplimiento del } \\
\text { tiempo de retiro }\end{array}$ & $\begin{array}{c}\text { ct } 1=\text { acciones correctivas frente al incumplimiento } \\
\text { del tiempo de retiro } \\
\text { ct2= no acciones correctivas frente al } \\
\text { incumplimiento del tiempo de retiro }\end{array}$ \\
\hline Área de cuarentena & $\begin{array}{l}\text { cu1 }=\text { si área de cuarentena } \\
\text { cu2= no área de cuarentena } \\
\text { u3 }=\text { no ingreso de foráneos }\end{array}$ & $\begin{array}{l}\text { Tanque de almacenamiento de } \\
\text { agua }\end{array}$ & $\begin{array}{l}\operatorname{tag} 1=\text { Tanque de aguaen buenascondiciones } \\
\text { tag2 }=\text { Tanque de aguaen malas condiciones }\end{array}$ \\
\hline Asistencia técnica & $\begin{array}{l}\text { a1= recibe asistencia técnica } \\
a 2=\text { no recibe asistencia técnica }\end{array}$ & Clasificación de MV & $\begin{array}{c}\mathrm{cm} 1=\text { adecuadaclasificación de MV } \\
\mathrm{cm} 2=\text { =inadecuada clasificación de MV }\end{array}$ \\
\hline $\begin{array}{l}\text { Pisos, paredes y techos } \\
\text { debidamente acabados y con } \\
\text { superficies fáciles de limpiar y } \\
\text { desinfectar }\end{array}$ & $\begin{array}{c}\text { sf1 = Pisos, paredes y techos fáciles de limpiar } \\
\text { y desinfectar } \\
\text { sf2= Pisos, paredes y techos difíciles de limpiar } \\
\text { y desinfectar }\end{array}$ & $\begin{array}{l}\text { Almacenamiento de alimentos para } \\
\text { animales }\end{array}$ & $\begin{array}{c}\text { aaa1=adecuado almacenamiento de } \\
\text { concentrados } \\
\text { aaa2= inadecuado almacenamiento de } \\
\text { concentrados }\end{array}$ \\
\hline
\end{tabular}




\section{Utilización del cuarto del tanque de enfriamiento solo para los propósitos establecidos \\ Procedimiento de limpieza y desinfección (LD)}

Garantía de conservación de la leche a temperatura por debajo de $6^{\circ} \mathrm{C}$

Drenajes con sifón o trampa que impiden el acceso de plagas al áre

\section{Puertas cerradas en todo momento $y$ ventanas protegidas con angeos \\ Luz natural o artificial adecuada y bien distribuida, luz artificial con pantalla protectora en sala del tanque \\ Equipo para agua caliente \\ Equipo de refrigeración operado y mantenido en buen estado}

Ventilación adecuada

Ordeño en sitio apropiado y dedicado exclusivamente para este fin

Instalaciones de la sala de ordeño

Existe un procedimiento de LD para el sitio de ordeño

Zona de espera en condiciones de higiene adecuadas

Restricción a otros animales en la sala de ordeño

uso1= adecuad utilización del cuarto del tanque de enfriamiento

uso2= inadecuada utilización del cuarto del tanque de enfriamiento uso $3=$ no cuenta con tanque de enfriamiento

$$
\begin{gathered}
\mathrm{p} \mid 1=\text { existe protocolo de LD } \\
\mathrm{p} \mid 2=\text { no existe protocolo de LD } \\
\mathrm{pl} 3=\text { no se necesita protocolo de LD }
\end{gathered}
$$

$c 1=$ se conserva la leche a temperatura por debajo de $6^{\circ} \mathrm{C}$ debajo de $6^{\circ} \mathrm{C}$

c3= no hay tanque de enfriamiento y la leche se entrega directamente al intermediario

np1=existencia de sifón o trampa que impida acceso de plagas $\mathrm{np} 2=$ ausencia de sifón 0 trampa que impida el acceso de plagas

ppv1= protección de puertas y ventanas en sala de ordeño

$$
\text { sala de ordeño }
$$

11 = Buena iluminación e sala de tanque $12=$ Mala iluminación en sala de tanque $13=$ No necesita iluminación por no tener sala de tanque

ea1 $=$ Existe equipo para agua caliente ea2=no Existe equipo para agua caliente ea3= No requiere de equipo para agua caliente

me1= Equipo de refrigeración en buen estado me3=Ausencia de equipo de refrigeración
mén
2= No hay procedimiento de LD para sitio de ordeño

lo3= Procedimiento de LD innecesario en potrero

v1=ventilación adecuada v2= ventilación inadecuada

$3=$ no se cuenta con ventilación porque no hay tanque de enfriamiento

pel $1=$ Tanque de enfriamiento con planta electrica

pel2= Tanque de enfriamiento sin planta

pel3 $=$ No se necesita planta electrica por no tener planta electrica

$s 1=$ Ordeño en sitio apropiado y dedicado

exclusivamente para este fin s2=Ordeño en para este fin

\section{is $1=$ Sala de ordeño en buenestado \\ is $2=$ Sala de ordeño en mal estado}$$
\text { is } 3=\text { Ordeño en potrero }
$$

\section{$\mid z 1=$ Adecuadas condiciones de higiene en zona de espera} ordeño

rs2=no restriccion a otros animales en sala de ordeño
Iz2= Inadecuada higiene en zona de espera.

rs1= Restriccion a otros animales en sala de animales

c2=no se conserva la leche a temperatura por Notificación de efectos indeseables

inv1 = existe inventario de MVB

iv2=no existe inventario de MVB

ia1= hay inventario de alimentos para animales

ia2= no hay inventario de alimentos para animales

ead $1=$ se notifican efectos indeseables 0 adversos

ead2=no se notifican efectos indeseables o

adversos

localización según el plan de ordenamiento territorial

po1= localización según POT po2=no localización según POT

Identificación de áreas

id1= hay identificación de áreas id2=no hay identificación de áreas

Instalaciones sanitarias

Manejo de guías sanitarias de movilización

isn $1=$ Presencia de instalaciones sanitarias isn2= Ausencia de instalaciones sanitarias

g1= Se manejan guías sanitarias de movilización g2= No se manejan guías sanitarias de movilización

ran1= Existe registro individual de cada animal ran $2=$ No existe registro individual de cada animal

Clasificación de basuras

$$
\text { cba1=Adecuada clasificación de basuras }
$$
cba2=no hay clasificación de basuras

Manejo de basuras y residuos peligrosos

rpa1 $=$ Adecuado manejo de residuos rpa2= Inadecuado manejo de residuos

Acciones para el control de plagas

cpa1 $=$ Se implementanacciones para el control de plagas

cpa2=no se implementanacciones para el control de plagas

Manejo y disposición de estiércol en instalaciones

deia1 = Adecuado manejo del estiércol deia2= Inadecuado manejo del estiérco deia3=animales fuera de instalaciones

Estado sanitario del personal de ordeño y examen médico

esoa $1=$ Personal con adecuado estado sanitario esoa2= Personal en inadecuado estado sanitario

Programa de capacitación os certificados de asistencia

capa $1=$ Certificados de asistencia a capacitaciones capa2 $=$ Ausencia de certificados de asistencia a capacitaciones

dta1= Cuentan con implementos de trabajo y dotación

Implementos de trabajo y dotación dotación

dotación 


\begin{tabular}{|c|c|c|c|}
\hline $\begin{array}{c}\text { Luz natural o artificial adecuada } \\
\text { y bien distribuida, la luz artificial } \\
\text { con pantalla protectora en sala de } \\
\text { ordeño }\end{array}$ & $\begin{array}{c}\text { Izo1= Adecuada iluminacion en sala de ordeño } \\
\text { Izo2= Inaadecuada iluminacion en sala de } \\
\text { ordeño }\end{array}$ & Seguridad social & $\begin{array}{c}\text { sss1= Trabajadores afiliados a seguridad social } \\
\text { sss2= Trabajadores no afiliados a seguridad } \\
\text { social }\end{array}$ \\
\hline $\begin{array}{c}\text { Manejo de residuos sólidos y } \\
\text { líquidos sin riesgo para fuentes de } \\
\text { agua, ambiente y proliferación de } \\
\text { plagas }\end{array}$ & $\begin{array}{l}\mathrm{mr} 1=\text { Adecuado manejo de residuo } \\
\mathrm{mr} 2=\text { Inadecuado manejo de residuos }\end{array}$ & $\begin{array}{l}\text { Botiquín y al menos una persona } \\
\text { capacitada en primeros auxilios } \\
\text { (PA) }\end{array}$ & $\begin{array}{c}\text { bta1= Botiquín y personal capacitado en PA } \\
\text { bta2=Ausencia de Botiquín y personal capacitado } \\
\text { en PA }\end{array}$ \\
\hline $\begin{array}{l}\text { Procedimiento para rutina de } \\
\text { ordeño }\end{array}$ & $\begin{array}{c}\text { pro1=Existencia de procedimiento para rutina } \\
\text { de ordeño } \\
\text { pro2= Ausencia de procedimientos para rutina } \\
\text { de ordeño }\end{array}$ & $\begin{array}{c}\text { Protección de la leche, equipos y } \\
\text { utensilios (EU) }\end{array}$ & $\begin{array}{l}\text { pr1 }=\text { Proteccion a leche y EU } \\
\text { pr2= No protección a leche y EU }\end{array}$ \\
\hline $\begin{array}{l}\text { Lavado de manos y antebrazos } \\
\text { antes de iniciar la rutina de ordeño }\end{array}$ & $\begin{array}{l}\text { Im1= Adecuada higiene de ordeñadores } \\
\operatorname{Im} 2=\text { Inadecuada higiene de ordeñadores }\end{array}$ & $\begin{array}{l}\text { protección de superficies en } \\
\text { contacto con la leche que hayan } \\
\text { sido desinfectados }\end{array}$ & $\begin{array}{c}\text { prs1= desinfección de superficies en contacto } \\
\text { con eche } \\
\text { prs2= Inadecuada desinfección de superficies en } \\
\text { contacto con leche }\end{array}$ \\
\hline Despunte & $\begin{array}{c}\text { ds } 1=\text { Realiza despunte } \\
\text { ds } 2=\text { No realiza despunte }\end{array}$ & $\begin{array}{l}\text { Ordeño por separado de las vacas } \\
\text { que producen leche anormal }\end{array}$ & $\begin{array}{l}\text { (sv1= Ordeño separado de animales enfermos } \\
\text { sv2=No ordeño separado de animales enfermos }\end{array}$ \\
\hline $\begin{array}{l}\text { Pezones limpios al momento del } \\
\text { ordeño }\end{array}$ & $\begin{array}{l}\text { Ipz1= Pezones limpios al ordeño } \\
\text { Ipz2= Pezones no limpios al ordeño }\end{array}$ & $\begin{array}{l}\text { Materiales adecuados de equipos } \\
\text { y utensilios }\end{array}$ & $\begin{array}{c}\text { mu1 }=\text { Materiales adecuados mu2= Materiales } \\
\text { inadecuados }\end{array}$ \\
\hline Pezones desinfectados al ordeño & $\begin{array}{c}\text { dpz1= desinfeccion de pezones } \\
d p z 2=\text { no desinfeccion de pezones }\end{array}$ & $\begin{array}{l}\text { Única utilización y adecuado } \\
\text { almacenamiento de los filtros para } \\
\text { la leche }\end{array}$ & $\begin{array}{l}\text { fu1 }=\text { adecuado uso de filtros de leche } \\
\text { fu2= inadecuado uso de filtros de leche }\end{array}$ \\
\hline Secado de pezones & $\begin{array}{c}\text { spz1 }=\text { Secado de pezones } \\
\text { spz2= No secado de pezones }\end{array}$ & $\begin{array}{l}\text { Procedimientos de LD para } \\
\text { equipos y utensilios }\end{array}$ & $\begin{array}{c}\text { pleu1 }=\text { Existe procedimiento de LD de equipos y } \\
\text { utensilios } \\
\text { pleu2 }=\text { No existe procedimiento de LD de equipos } \\
\text { y utensilios }\end{array}$ \\
\hline Sellado de pezones & $\begin{array}{l}\text { sll1= Sellado de pezones } \\
\text { sll2= No sellado de pezones }\end{array}$ & $\begin{array}{l}\text { Registros de mantenimiento } \\
\text { preventivo del equipo de ordeño }\end{array}$ & $\begin{array}{l}\mathrm{rm} 1=\text { Registro de mantenimiento preventivo } \\
\mathrm{rm} 2=\text { no registro de mantenimiento preventivo }\end{array}$ \\
\hline Calidad del agua & $\begin{array}{l}\text { cag1 }=\text { Calidad del agua óptima } \\
\text { cag2 }=\text { Calidad del aguadeficient }\end{array}$ & & \\
\hline
\end{tabular}

son el uso del tanque de enfriamiento exclusivamente para almacenamiento de la leche, existencia de procedimientos para limpieza y desinfección de equipos, garantía de conservación de la leche por debajo de 6 ${ }^{\circ} \mathrm{C}$ y operación y estado de los equipos de refrigeración.

Se encontraron dos grupos de sistemas (Figura 1). Explotaciones en los que el tanque de enfriamiento puede ser usado para propósitos diferentes a los establecidos (uso2), donde faltan procedimientos de limpieza y desinfección (pl2), en los cuales no se conserva estrictamente la leche a temperaturas inferiores a $6{ }^{\circ} \mathrm{C}(\mathrm{C} 2)$ y que no garantizan el correcto estado y operación de los equipos de refrigeración (Me2).

En el extremo opuesto del eje, se ubicaron sistemas que no cuenta con tanque de enfriamiento de leche (Uso3), por lo tanto, la leche no es almacenada, sino entregada directamente al intermediario (c3), no se poseen protocolo de limpieza y desinfección (pl3), ni equipo de refrigeración (me3), agua caliente o planta eléctrica (ea3 y pel3) y no es un punto crítico la iluminación, ni la ventilación (I3 y v3), por no tener sala de tanque (I3)
El eje vertical (dimensión 2, la cual explica el restante $11,82 \%$ de la variabilidad de los datos), involucra variables que tienen que ver con un adecuado procedimiento de ordeño y condiciones de higiene durante la faena, que involucran tanto al operario, como al animal, en variables como la existencia de procedimientos para la rutina de ordeño, la higiene de las manos del operario y de los pezones de la vaca antes del ordeño, la realización de despunte y la restricción al ingreso de otras especies a la sala de ordeño.

En un grupo se encontraron sistemas en los cuales hay establecido un procedimiento para la rutina de ordeño (pro1), antes del ordeño se da adecuado lavado de manos y antebrazos ( $\operatorname{Im} 1)$, se realiza desinfección de pezones (dpz1), despunte (ds1) y en la sala de ordeño hay restricción al ingreso de otras especies (rs1).

Un segundo grupo involucró sistemas en los cuales no hay establecido un procedimiento de ordeño (pro2), no se garantiza adecuada higiene de manos de ordeñadores, ni de los pezones de los animales (Im2 y dpz2) y hay ingreso de otros tipos de animales a la sala de ordeño (rs2) 


\section{Two panel biplot}
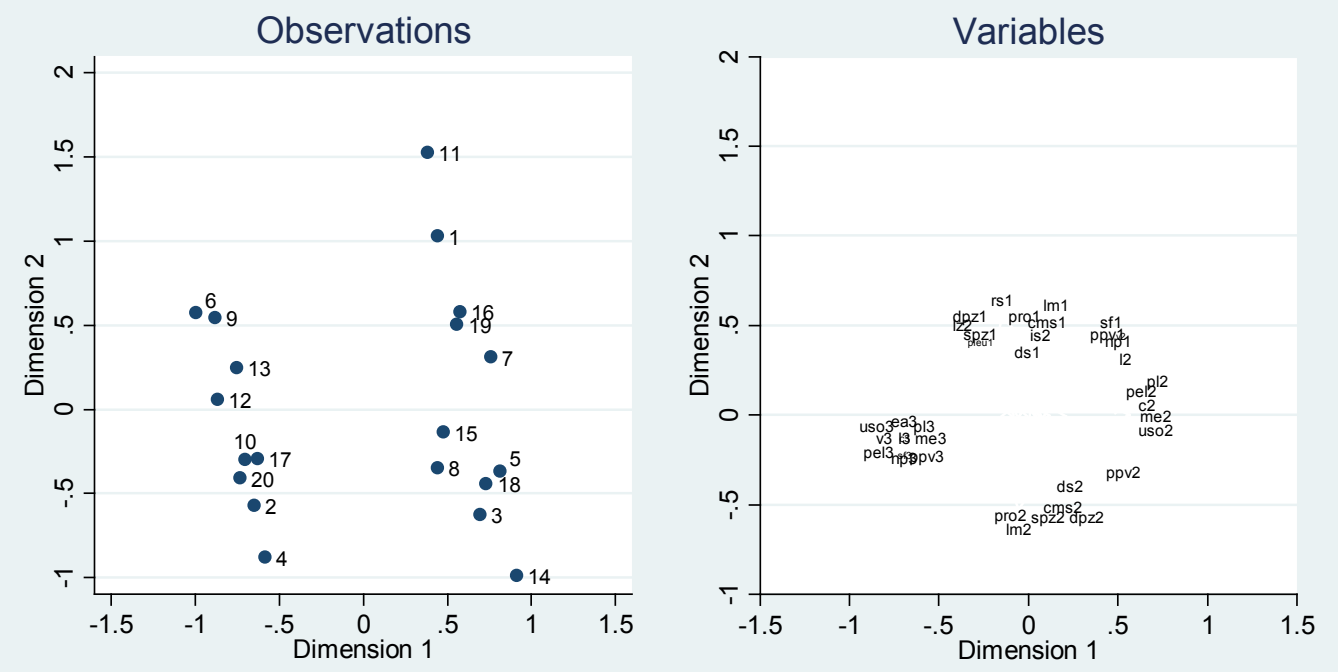

Figura 1. Plano factorial en el que se ubican las observaciones y las variables según el análisis de correspondencia múltiple

Con respecto a las observaciones, en el plano factorial, se formaron dos grupos diferenciados, el primero, conformado por hatos con ordeño manual, en potrero o en sala, debido a la similitud de ítems que los relaciona y la otra agrupación hace referencia a los hatos con ordeño mecánico, en sala o en potrero, quienes comparten similitudes como la tenencia de equipos de ordeño, cuarto de para tanque de enfriamiento, tanque de enfriamiento y todos los protocolos y registros relacionados con ello.

\section{CONCLUSIÓN}

Existe un adecuado desempeño en áreas que involucran el ordeño y el bienestar animal, pero son deficientes en áreas como el control de medicamentos veterinarios e insumos agropecuarios, los registros, la seguridad del personal y primeros auxilios, por lo que se asume que falta capacitación al respecto.

Las BPG son explicadas y hay alta influencia de variables que tienen que ver directamente con la calidad del producto final, en relación al estado de equipos y la higiene durante la rutina de ordeño; posiblemente otras variables no incluidas en dicha lista de verificación puedan explicar mejor la variabilidad de los datos y una vez evaluada dicha herramienta, fundamental para el diagnóstico, sea necesaria la implementación y posterior evaluación y seguimiento a planes de mejoramiento de los sistemas productivos.

\section{REFERENCIAS}

CARULLA, J.; ORTEGA, E. 2016. Sistemas de producción lechera en Colombia: retos y oportunidades. Archivos Latinoamericanos de Producción Animal. Volumen 24(2):2016.

INSTITUTO COLOMBIANO AGROPECUARIO (ICA). 2008. Resolución 3278 de 2008. Diario oficial número 47.130. Por la cual se establece la Expedición de Guías Sanitarias de Movilización Interna mediante el Sistema Nacional Web de Movilización y Comercialización Ganadera.

INSTITUTO COLOMBIANO AGROPECUARIO ICA. 2013. Guías Sanitarias de Movilización Interna - GSMI. Subgerencia de protección animal. En: http://www.ica.gov.co/getattachment/e463cf32-2320-4ea5-8b9058be053ecea6/GSMI-Guias-Sanitarias-de-Movilizacion-Interna.aspx 
INSTITUTO COLOMBIANO AGROPECUARIO (ICA). 2015. Resolución número 001332 del 6 de mayo de 2015. "Por medio de la cual se modifica la Resolución 1513 del 15 de julio de 2004". En: http://legal.legis.com.co/docum ent?obra=legcol\&document=legcol_fdac62a3c8e34c7e9a5a5f470c1c09ba Fecha de consulta: 01-04-2017

MADR-MINISTERIO DE AGRICULTURA Y DESARROLLO RURAL. 2012. Resolución número 000017. Por la cual se establece el sistema de pago de leche cruda al proveedor. En: http://confia.com.co/index.php/normas-y-decretos/ item/40-resolucion-000017-de-2012-leche-cruda-pago-por-calidad Fecha de consulta: 22-03-17.

MADR-MINISTERIO DE AGRICULTURA Y DESARROLLO RURAL. 2013. Sistema de información geográfica municipal. Santa Rosa de Cabal- Risaralda. Oferta agropecuaria. En: http://bibliotecadigital.agronet.gov.co/ bitstream/11348/3728/2/SIG-ACTUALIZACION_SANTA\%20ROSA\%20DEL\%20CABAL_RISARALDA.pdf. Fecha de consulta: 23-03-17

MINISTERIO DE PROTECCIÓN SOCIAL. 2006. Decreto número 616 de 2006. Por el cual se expide el Reglamento Técnico sobre los requisitos que debe cumplir la leche para el consumo humano que se obtenga, procese, envase, transporte, comercializa, expenda, importe o exporte en el país. En: http://www.ica.gov.co/getattachment/15425e0f81fb-4111-b215-63e61e9e9130/2006D616.aspx Fecha de consulta 22-03-17.

MINISTERIO DE SALUD Y PROTECCIÓN SOCIAL. 2013. Resolución 2674 de 2013. Por la cual se reglamenta el Artículo 126 del Decreto-ley 019 de 2012 y se dictan otras disposiciones.

MUR, A.; MOLANO, J. 2016. Buenas prácticas ganaderas: ¿Mito, inconsciencia ganadera o falta de apoyo estratégico? FAGROPEC. 8(1):12- 8.

VALENCIA, M.; AGUILAR, O.; RODRIGUEZ, A. 2012. Caracterización de los pacientes en una consulta de hipertensión arterial de un municipio de Risaralda, Colombia, 2005-2012. Revista médica Risaralda. 18(2).

VILLOCH, A. 2010. Good agricultural practices for the production of milk. His objetives and relation with the codes of hygienic practice. Revista Salud Animal. 32 (3):2010. 